\title{
Acoustic Emission Monitoring of Degradation in Plain Weave CFRP Composite Laminate
}

\author{
Su-Ping Ai, Chang-Hang Xu, Guo-Ming Chen, Guo-Rui Li \\ College of Mechanical and Electronic Engineering, \\ China University of Petroleum (East China), \\ Qingdao, China
}

\begin{abstract}
The failure process on the plain weave CFRP composite laminate under three-point bending test was monitored by acoustic emission (AE). Failure process was divided into stage I, II, and III based on the energy distribution along with the mechanical results. Moreover, various failure modes in every stage were identified by RAAF method. Damage evolution for each failure mode was further quantified and evaluated through b-value method. Results reveal that no damage can be observed in stage I, and the dominant failure mode in stage $\mathrm{II}$ is matrix cracking, while stage III, where the specimen actually fractured, failure modes such as matrix cracking, fibre breakage and delamination arise sequentially. It is shown that AE monitoring can be applied for characterizing and evaluate damage in plain weave composite laminate.
\end{abstract}

Keywords- plain weave; CFRP; acoustic emission; parameter analysis

\section{INTRODUCTION}

Carbon fibre reinforced composite (CFRP) has been applied in various fields such as aerospace engineering and automotive. Plain weave, with warp ( $x$-direction yarns) and fill ( $y$-direction yarns) threads crossing alternately in laminates to offer higher impact resistance and damage tolerance than traditional fibre reinforcement, was widely used in composite manufacturing as the most common fibre reinforcement type in last three decades. During service, composite structures may suffer external loads which gradually decrease the strength and stiffness and finally lead to some damage affects such as matrix cracking, fibre breakage, and delamination. Therefore, the ability to monitor the failure process in composite materials is essential.

Acoustic emission (AE), which is based on detecting the stress waves induced by external force, was confirmed as a suitable in situ healthy monitoring method. A key issue of AE monitoring is to build the correlation between the detected AE signals and damage evolution. Various AE signal analysis methods in damage evolution were developed by previous researchers. Loutas et al. [1] classified AE signals recorded from woven carbon/carbon composites failure process to four categories by unsupervised pattern recognition algorithms. A function combined mechanical and acoustic energy was performed by Bakhtiary Davijanito to measure the delamination failure in 2D woven composites with different weave architectures[2]. Tensile-tensile fatigue performance of woven fabric composites was fully investigated and the failure behavior was classified into four stages through AE amplitude analysis by Pandita [3]. These studies have demonstrated the potential of AE technique in monitoring damage evolution in composite materials.

Building on these advancements, the objective of this work is to interpret AE signals to determine and evaluate damage behaviors during the three-point bending test in plain weave CFRP composite laminate. Firstly, mechanical results along with AE observations of energy distribution were discussed. The failure process was divided into three stages based on the discussion results. Damage information was further analysed by RA-AF method and b-value method.

\section{AE PARAMETER ANALYSIS}

AE parameter analysis [4], as an essential method for identifying damage types, uses the typical features such as amplitude, energy, rising time, duration time and frequency. A typical AE signal is shown in Figure 1, where the useful parameters are defined. The relationship between RA (rising time divided by the maximum amplitude) and AF (counts divided by duration time) values was used to differentiate signals from tensile and shear cracks in some researches [5, 6]. Signal that has low average frequency and high RA value can be regarded as tensile type cracks. While signal with the high average frequency and low RA value is regarded as shear type cracks (seen in Figure 2).

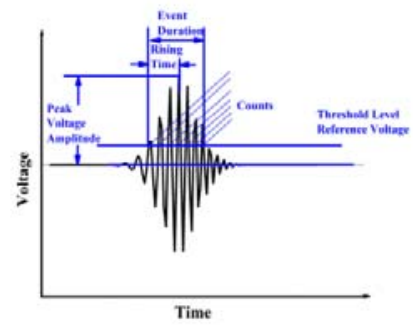

Figure 1. Schematic showing Relationship between AF and RA value. 


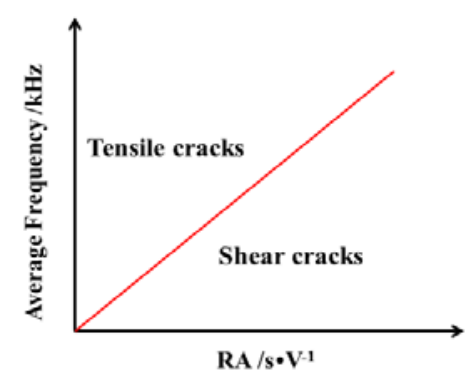

Figure 2. Acoustic emission waveform and basic.

b-value method was first widely used in seismology field to express the relationship between the magnitude and total number of earthquakes. It is also a possible indicator to evaluate size distribution of $\mathrm{AE}$ sources by applying a correction factor to the AE amplitudes. b-value defined by Aki's method, gives each recorded AE event an equal weighting by applying the maximum likelihood method[7], can be computed as follows:

$$
b=\left(20 \times \log _{10} e\right) \div\left(\bar{a}-a_{0}\right)
$$

where $e=2.71828, b$ is the $b$-value, $\bar{a}$ is the mean amplitude and $a_{0}$ is the threshold amplitude of the AE events that are recorded for a detected area over a period of time.

\section{THE EXPERIMENTAL SETUP}

\section{A. Materials}

The experimental work was carried out on plain weave CFRP composite laminate with constant thickness. The fibre of plain weave fabric was made of carbon T300-3K produced by Toray Industries, Inc. Twelve layers of bidirectional plain weave carbon fabrics $\left[0^{\circ} / 90^{\circ}\right] \mathrm{s}$ were stacked. The size of the composite laminate is $250 \mathrm{~mm} \times$ $100 \mathrm{~mm} \times 2 \mathrm{~mm}$.

\section{B. Loading and Acoustic Emission}

The three-point bending test was performed on the specimen using a Universal Machine INSTRON under monotonous loading until the moment of load drop. The load was controlled through the displacement control mode with the fixed crosshead speed of $2 \mathrm{~mm} / \mathrm{min}$. Figure 3 presents the configuration of the three-point bending test.

Concerning AE monitoring, a four-channel AE system (PCI-2 by PAC), with a sampling rate of $5 \mathrm{MHz}$, was used to record the $\mathrm{AE}$ activities during the fatigue loading test. Four broadband sensors were placed on the upper surface of the specimen by adhesive tape with a thin layer of silicone grease which has proven to be a good couplant, shown in Figure 3. The 2/4/6 preamplifiers with gain of $40 \mathrm{~dB}$ and were used to pre-amplify the acquired $\mathrm{AE}$ signals.

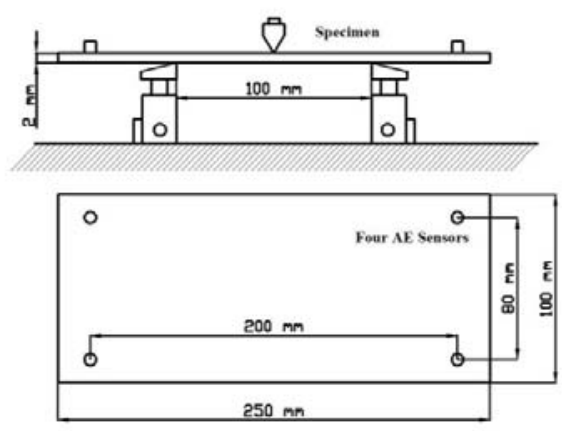

Figure 3. Specimen laminate geometry and the three-point bending test configuration.

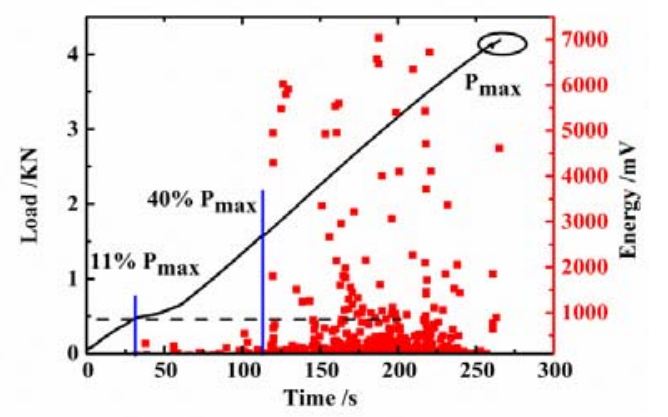

Figure 4. Curves of load and AE energy versus time for plain weave CFRP composite laminate under three-point bending test.

\section{RESULTS AND DISCUSSION}

\section{A. Crack Development and Classification}

The AE energy, which is proportional to the released energy at the fracture incidents, has been used in damage characterization in similar studies [7, 8]. In order to illustrate the relationship between $\mathrm{AE}$ results and the mechanical properties, a typical case of load and AE energy with time were plotted in Figure 4. The AE energy is shown to change with increased external load. Three stages of failure process associated with different failure behaviors can be identified.

Stage I (from beginning to $30 \mathrm{~s}$ ): In the initial phase, the load increases linearly with time until the load reaches $11 \%$ of the maximum load. In this phase no obvious AE signal can be detected. This means that there is no damage in the area monitored with $\mathrm{AE}$. 


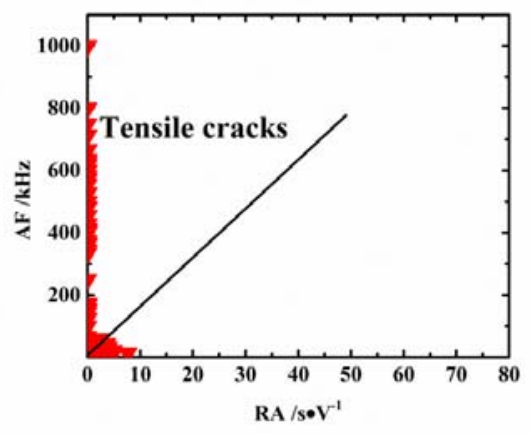

Figure 5. RA and AF value history for stage II.

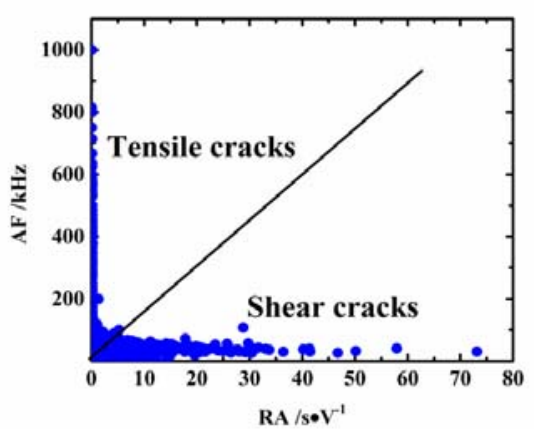

Figure 6. RA and AF value history for stage III.

Stage II (from 30 to 118 s): During this stage, a change in the slope can be observed. The load increases under an exponential law. AE activities with low energy (less than $1000 \mathrm{mV}$ ) can be obtained. It can be concluded that damage starts at load values close to $0.5 \mathrm{KN}$. The RA versus $A F$ in this stage was plotted in Figure 5 . It can be seen that most date points have various AF and low RA values (the range for $\mathrm{AF}$ is between 0 and $1000 \mathrm{kHz}$, and is less than $10 \mathrm{~s} / \mathrm{V}$ for RA). Therefore, based on Figure 2, this indicates that the main type of crack is pure tensile. Matrix cracking, as the first form of damage in laminates, are intralaminar or ply cracks that traverse the thickness of the ply and propagate parallel to the fibres in that ply. Such damage mode can lead to tensile cracks. In general, during this period, the main failure mode is the matrix cracking within plies with lower energy caused by the high load concentration under the loading nose.

Stage III (from $118 \mathrm{~s}$ to end): The rise of AE energy occurs at $1.6 \mathrm{kN}$, which is about $40 \%$ of the maximum load. As the load increases, the AE activities with high energy (greater than $1000 \mathrm{mV}$ ) become significant. Moreover, the amount of AE events with lower energy is larger compared with the previous stage. Figure 6 depicts the relationship between RA and AF in stage III. In this stage, apart from the data points which represents tensile mode cracks, some data points with a wider range of RA values from 0 to $74 \mathrm{~s} / \mathrm{V}$ and much lower AF (less than 200 $\mathrm{kHz}$ ) which indicates the shear mode cracks are observed. During this stage, matrix cracks nucleate, some tend to deviate to boundary layer and cause delamination at ply boundary in the weak bonding interface. In addition, some fibre breakage events are vulnerable to pull out and break due to the crack grow into fibre yarns. These failure modes can cause the shear mode cracks in this stage.

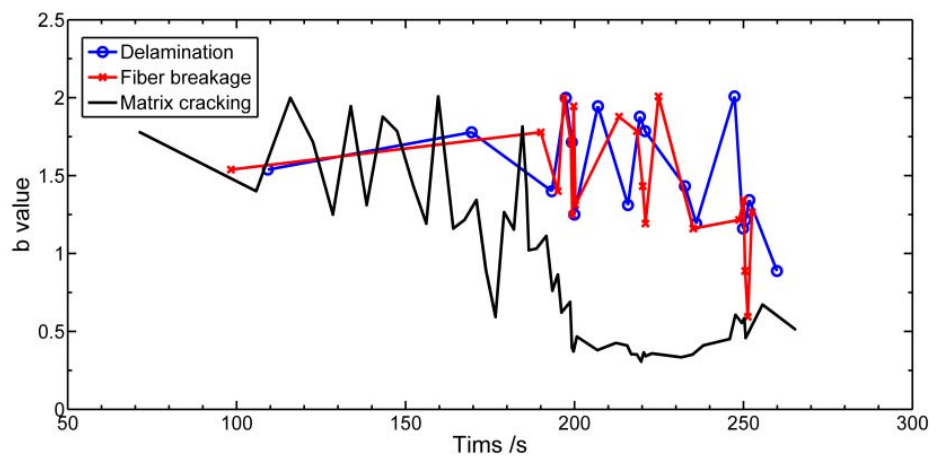

Figure 7. Variation of b-value with time for matrix cracking, fibre breakage and delamination.

\section{B. Damage Level evaluation}

In order to quantify and evaluate damage level, bvalue method was utilized in this work. The b-value method introduced in this work is based on using amplitude distribution of each $\mathrm{AE}$ signal to evaluate the damage level for every failure mode. The corresponding lower and upper limiting ranges of $\mathrm{AE}$ amplitude were gained from some previous studies[9], which is defined as 45-70 dB, 70-80 dB, and 80-98 dB for matrix cracking, delamination, and fibre breakage, respectively. The b- value for each failure mode gives valuable information about the changes in stress-induced cracks. The occurrence of initiation point indicates the stress reaching the value which can cause the failure mode. b-value tends to increase when microcracks is dominant, whereas tends to decrease when macrocracks occur more frequently. The b-value of individual subsets was calculated by equation (1) for every 50 events till the end of load time. Results are shown in Figure 7.

For matrix cracking, the b-value is 1.78 when it initiates on the surface of specimen's central area. Matrix 
cracks propagate and expand as the load increases. The bvalue declines and reaches the minimum value at $220 \mathrm{~s}$. With further increase in load, the b-value increases slightly, which may cause by the transition from formation to growth stage of the newly formed matrix cracks. In the case of fibre breakage, some individual fibre breakage starts around $100 \mathrm{~s}$ with the b-value about 1.53 , which is smaller than the matrix cracking. The bvalue shows fluctuation during early loading stage. Around the point of failure, a rapid decrease indicating that more fibre breakage damage occurs can be observed. The lowest b-value is recorded at $250 \mathrm{~s}$. The change of $b$-value for delamination follows the same tendency as that for fibre breakage, while shows $10 \mathrm{~s}$ later than that for fibre breakage. From 247 s, the b-value begins to fall. The minimum value of $b$ curve is exhibited at point of failure. The lower b-value indicates more severe damage, it can be implied that most delamination damage shows in the instant of failure point. The b-value analysis results can clearly indicate the onset of unstable cracking as well as crack coalescence which leads to final failure of the specimen.

\section{CONCLUSIONS}

In this paper, the $\mathrm{AE}$ technique was applied to monitor the damage evolution of plain weave CFRP composite laminate under three-point bending test. The following conclusions were made:

(1) The failure process contains three key stages. No failure behavior can be detected in the first stage. Failure performance is initiated by transverse cracks nucleation in matrix from second stage. The cracks then expand into fibre yarn or along the ply caused delamination as well as fibre breakage in last stage.

(2) In addition, for better visualization of the damage evolution for the last stage of which accumulated damage involves multiple failure modes such as matrix cracking, fibre breakage and delamination, the use of b-value is recommended. The b-value is found to be sensitive to the damage evolution, since the b-value curves of various failure modes gradually change as the damage process. It can be seen that the severe damage in matrix cracking occurs at around $200 \mathrm{~s}$. After the failure of matrix cracking, large scale damage in fibre breakage and delamination appears at the stress close to the failure of specimen.

(3) AE method is a vital application for monitoring the stability and integrity of composite structures at condition.

\section{REFERENCES}

[1] Loutas TH, Kostopoulos V, Health monitoring of carbon/carbon, woven reinforced composites: damage assessment by using advanced signal processing techniques. Part II: Acoustoultrasonics monitoring of damage development. Composites Science and Technology, 69(2), pp. 273-283, 2009.

[2] Bakhtiary Davijani AA, Hajikhani M, Ahmadi M, Acoustic emission based on sentry function to monitor the initiation of delamination in composite materials. Materials and Design, 32(5), pp. 30593065, 2011.

[3] Pandita SD, Huysmans G, Wevers M, Verpoest I, Tensile fatigue behaviour of glass plain-weave fabric composites in on- and offaxis directions. Journal of Composites Part A: Applied Science and Manufacturing, 32(10), pp. 1533-1539, 2001.

[4] Elfergani HA, Pullin R, Holford KM, Damage assessment of corrosion in prestressed concrete by acoustic emission. Construction and Building Materials, 40, pp. 925-933, 2013.

[5] Aggelis DG, Mpalaskas AC, Matikas TE, Investigation of different fracture modes in cement-based materials by acoustic emission. Cement and Concrete Research, 48, pp. 1-8, 2013.

[6] Aldahdooh MAA, Muhamad Bunnori N, Crack classification in reinforced concrete beams with varying thicknesses by mean of acoustic emission signal features. Construction and Building Materials, 45, pp. 282-288, 2013.

[7] Sagar RV, Prasad BKR, Kumar SS, An experimental study on cracking evolution in concrete and cement mortar by the b-value analysis of acoustic emission technique. Cement and Concrete Research, 42, pp. 1094-1104, 2012.

[8] Rouchier S, Foray G, Godin N, Woloszyn M, Roux J, Damage monitoring in fibre reinforced mortar by combined digital image correlation and acoustic emission. Construction and Building Materials, 38, pp. 371-380, 2013.

[9] Mechraoui S, Laksimi A, Benmedakhene S, Reliability of damage mechanism localisation by acoustic emission on glass/epoxy composite material plate. Composite Structures, 94(5), pp. 14831494, 2012 